\title{
APPROXIMATION BY POLYNOMIALS WITH LOCALLY GEOMETRIC RATES
}

\author{
K. G. IVANOV, E. B. SAFF AND V. TOTIK
}

(Communicated by Irwin Kra)

\begin{abstract}
In contrast to the behavior of best uniform polynomial approximants on $[0,1]$ we show that if $f \in C[0,1]$ there exists a sequence of polynomials $\left\{P_{n}\right\}$ of respective degree $\leq n$ which converges uniformly to $f$ on $[0,1]$ and geometrically fast at each point of $[0,1]$ where $f$ is analytic. Moreover we describe the best possible rates of convergence at all regular points for such a sequence.
\end{abstract}

\section{INTRODUCTION}

Our paper is related to the fact that best polynomial approximants are very far from giving good approximation on subsets of the original set. In fact, let $\|\cdot\|_{[0,1]}$ denote the sup norm on $[0,1]$, let $f$ be continuous and real-valued on $[0,1]$, and $Q_{n}=Q_{n}(f)$ be the best uniform approximant to $f$ out of $\Pi_{n}$, the set of polynomials of degree at most $n$. A celebrated result of Kadec [2] says that the extremal points of $\left\{\left|f-Q_{n}(f)\right|\right\}_{n=0}^{\infty}$ are dense on $[0,1]$, and so on any subinterval $I \subseteq[0,1]$ the approximation given by $\left\{Q_{n}(f)\right\}_{n=0}^{\infty}$ (considering the whole sequence) is not better than on the whole interval $[0,1]$, no matter how smooth $f$ is on $I$.

In [3] it was shown that the situation radically changes if one considers near best approximants instead of best ones. For example, when $f$ is piecewise analytic on $[0,1]$ and otherwise $k$-times continuously differentiable at the

Received by the editors June 22, 1988.

1980 Mathematics Subject Classification (1985 Revision). Primary 41A25, 41A10.

Key words and phrases. Algebraic polynomials, approximation of functions, rate of decrease.

The research of the first author was supported, in part, by Contract No. 50, Committee for Science, Bulgaria. This work was done while he visited the University of South Florida, Tampa, Florida.

The research of the the second author was supported, in part, by the National Science Foundation under grant DMS-862-0098.

The research of the third author was supported, in part, by the Hungarian National Science Foundation for Research, Grant No. 1157. This work was done while he visited the University of South Florida, Tampa, Florida. 
non-regular points it was shown that for each $\beta>1$ there are constants $C$, $c>0$ and polynomials $p_{n} \in \Pi_{n}, n=1,2, \ldots$, such that

$$
\left|f(x)-p_{n}(x)\right| \leq \frac{C}{n^{k+1}} \exp \left(-c n[d(x)]^{\beta}\right), \quad x \in[0,1],
$$

where $d(x)$ measures the distance from $x$ to the nearest non-regular point of $f$. It was also shown that a similar estimate with $\beta=1$ is, in general, impossi= ble. These polynomials $p_{n}$, unlike the polynomials of best approximation, yield geometric convergence on (closed) intervals of analyticity even though $\left\{E_{n}(f)\right\}$, $E_{n}(f):=\left\|f-Q_{n}(f)\right\|_{[0,1]}$, has order only $\left\{n^{-k-1}\right\}$.

The problem whether similar results hold for more general sets of functions (not just for piecewise analytic ones) has however remained open. To be more precise we ask the following: let $f \in C[0,1]$ be analytic on the (relative to $[0,1])$ open subset $D$ of $[0,1]$. Is it possible to find polynomials $P_{n} \in \Pi_{n}$, $n=0,1, \ldots$, such that

$$
\left\|f-P_{n}\right\|_{[0,1]} \rightarrow 0 \quad \text { as } n \rightarrow \infty,
$$

and at every point of $D$ we have geometric convergence, i.e.

$$
\varlimsup_{n \rightarrow \infty}\left|f(x)-P_{n}(x)\right|^{1 / n}<1, \quad x \in D ?
$$

We will show that this is always possible and describe the behavior of the lefthand side of (1) which is, in a certain sense, best possible.

We will assume that $D$ is the exact set of analyticity, i.e. $D$ contains every regular point of $f$. For $x \in[0,1]$ let $d(x)$ be the distance from $x$ to the nearest singularity of $f$, where $f$ is considered to be extended to the complex plane and we also count the singularities outside $[0,1]$. In other words, $d(x)$ is the largest radius such that the Taylor expansion of $f$ about $x$ converges in $\{z \in \mathbf{C}:|z-x|<d(x)\}$. Of course, if $x$ is not a regular point of $f$, then $d(x)=0$.

If $d(x)>0$ for every $x \in[0,1]$, i.e. if $f$ is analytic on $[0,1]$, then the best uniform approximants converge geometrically to $f$ and so in what follows we assume that $f$ has a singularity somewhere on $[0,1]$.

Theorem 1. Suppose that $\beta>1$ and $f \in C[0,1]$ has a singularity on $[0,1]$. There are polynomials $P_{n} \in \Pi_{n}, n=0,1, \ldots$, such that

$$
\left\|f-P_{n}\right\|_{[0,1]} \rightarrow 0 \quad \text { as } n \rightarrow \infty \text {, }
$$

and for $x \in[0,1]$

$$
\left|f(x)-P_{n}(x)\right| \leq C_{f, x} \exp \left(-c n[d(x)]^{\beta}\right),
$$

where $c>0$ is an absolute constant and the constant $C_{f, x}$ is bounded for $x$ in any compact subset of $D$.

Next we show that Theorem 1 is best possible in the sense that (3) with $\beta=1$ is, in general, impossible. 
Theorem 2. There are no positive constants $C_{x}, x \in[-1,1]$, and $c>0$ such that $C_{x}, x \in D$, are bounded for every compact subset $D$ of $[-1,1]$ not containing the origin and for every $n$ there are polynomials $P_{n} \in \Pi_{n}$ with

$$
|| x\left|-P_{n}(x)\right| \leq C_{x} \exp (-c n|x|), \quad x \in[-1,1] .
$$

For convenience here the basic interval $[0,1]$ has been replaced by $[-1,1]$. Note that then for $f(x)=|x|$ we have $d(x)=|x|$.

Finally, we also show that Theorem 1 cannot be sharpened by putting a constant $C_{f}$ into (3)-the constant must depend on $x$, even allowing $c$ and $\beta$ to depend on $f$.

Theorem 3. There exists $f \in C[0,1]$ such that for no constants $\beta, C, c>0$ can one find polynomials $P_{n} \in \Pi_{n}, n=1,2, \ldots$, with the property

$$
\left|f(x)-P_{n}(x)\right| \leq C \exp \left(-c n[d(x)]^{\beta}\right), \quad n=1,2, \ldots, \quad x \in[0,1] .
$$

\section{Proof of Theorem 1}

It is enough to prove the theorem for $\beta \in(1,3 / 2]$ because $d(x) \leq 1$. Also it is enough to prove the theorem for $n \geq N_{0}$ because afterward we can increase $C_{f, x}$ so that (3) will be fulfilled for any natural number $n$. In the beginning $n$ is arbitrary and only when necessary we place restrictions on $N_{0}$.

For fixed $n \geq 1$ we set $\tilde{d}(x):=d(x)+n^{-1 / \beta}$ and define the points $0=$ $x_{0}<x_{1}<\cdots<x_{m}<x_{m+1}=1$ by $x_{0}:=0, x_{k+1}:=x_{k}+(1 / 40) \tilde{d}\left(x_{k}\right)$ whenever this defines a number $x_{k+1}$ with $x_{k+1}+(1 / 40) \tilde{d}\left(x_{k+1}\right) \leq 1$; in the opposite case, which occurs, say, for $k=m$, we set $x_{k+1}=x_{m+1}:=1$.

From the definition of $d(x)$ we have

$$
\left|\tilde{d}(x)-\tilde{d}\left(x_{k}\right)\right|=\left|d(x)-d\left(x_{k}\right)\right| \leq\left|x-x_{k}\right|,
$$

and so for $x \in\left[x_{k}, x_{k}+(1 / 40) \tilde{d}\left(x_{k}\right)\right], 0 \leq k \leq m$,

$$
\frac{39}{40} \tilde{d}\left(x_{k}\right) \leq \tilde{d}(x) \leq \frac{41}{40} \tilde{d}\left(x_{k}\right) .
$$

This easily implies that for each $k=0, \ldots, m$,

$$
\left|x_{k+1}-x_{k}\right| \leq \frac{1}{19} \tilde{d}\left(x_{k}\right),
$$

and thus

$$
\frac{18}{19} \tilde{d}\left(x_{k}\right) \leq \tilde{d}(x) \leq \frac{20}{19} \tilde{d}\left(x_{k}\right), \quad x \in\left[x_{k}, x_{k+1}\right] .
$$

We claim that

$$
\left[x_{k-2}, x_{k+3}\right] \subset\left[x-\frac{\tilde{d}(x)}{4}, x+\frac{\tilde{d}(x)}{4}\right], \quad x \in\left[x_{k}, x_{k+1}\right]
$$


(for definiteness set $x_{-2}=x_{-1}=0$ and $x_{m+2}=x_{m+3}=1$ ). In fact, we get from (5) and (6) that

$$
\begin{aligned}
x-\frac{\tilde{d}(x)}{4} & \leq x_{k}+\frac{1}{19} \tilde{d}\left(x_{k}\right)-\frac{1}{4} \cdot \frac{18}{19} \tilde{d}\left(x_{k}\right) \\
& \leq\left[x_{k-1}+\frac{1}{19} \tilde{d}\left(x_{k-1}\right)\right]-\frac{7}{38} \cdot \frac{18}{19} \tilde{d}\left(x_{k-1}\right) \\
& \leq x_{k-2}+\frac{1}{19} \tilde{d}\left(x_{k-2}\right)+\frac{1}{19} \cdot \frac{20}{19} \tilde{d}\left(x_{k-2}\right)-\frac{7}{38}\left(\frac{18}{19}\right)^{2} \tilde{d}\left(x_{k-2}\right) \\
& \leq x_{k-2},
\end{aligned}
$$

and similarly

$$
\begin{aligned}
x+\frac{\tilde{d}(x)}{4} & \geq x_{k}+\frac{1}{4} \cdot \frac{18}{19} \tilde{d}\left(x_{k}\right) \geq x_{k}+\frac{1}{19}\left[1+\frac{20}{19}+\left[\frac{20}{19}\right]^{2}\right] \tilde{d}\left(x_{k}\right) \\
& \geq x_{k}+\frac{1}{19}\left(\tilde{d}\left(x_{k}\right)+\tilde{d}\left(x_{k+1}\right)+\tilde{d}\left(x_{k+2}\right)\right) \\
& \geq x_{k+3} .
\end{aligned}
$$

By [1, Theorem 3] there exist two absolute constants $C_{1}, c_{1}$ with $c_{1} \leq 1$ such that for every $n$ there exists a polynomial $\chi_{n}^{*}=\chi$ of degree at most $n / 2$ such that on $[-1,1]$ the polynomial $\chi$ is monotone increasing, satisfies $0 \leq \chi \leq 1$ there and, with $\gamma:=(1+\beta) / 2>1$,

$$
\left|\chi(x)-\frac{1+\operatorname{sign} x}{2}\right| \leq C_{1} \exp \left(-c_{1} n|x|^{\gamma}\right), \quad x \in[-1,1],
$$

holds. With this $\chi_{n}^{*}=\chi$ ( $n$ is fixed) we define

$$
\begin{aligned}
& \chi_{0}(x):=1-\chi\left(x-x_{1}\right), \\
& \chi_{j}(x):=\chi\left(x-x_{j}\right)-\chi\left(x-x_{j+1}\right), \quad j=1, \ldots, m-1,
\end{aligned}
$$

and

$$
\chi_{m}(x):=\chi\left(x-x_{m}\right)
$$

Clearly we have

$$
\sum_{j=0}^{m} \chi_{j}(x)=1,
$$

and $0 \leq \chi_{j}(x) \leq 1$ on $[0,1]$. Furthermore, $(1+19 / 18)^{-\gamma}>1 / 4$ gives

$$
\begin{aligned}
\chi_{j}(x) & \leq 2 C_{1} \exp \left(-c_{1} n \min \left\{\left|x-x_{j}\right|^{\gamma} ;\left|x-x_{j+1}\right|^{\gamma}\right\}\right) \\
& \leq 2 C_{1} \exp \left(-\frac{1}{4} c_{1} n\left|x-x_{j}\right|^{\gamma}\right)
\end{aligned}
$$

provided $x \in[0,1] \backslash\left[x_{j-1}, x_{j+2}\right]$. This "partition of unity" will be used together with local best approximants to produce the required polynomial of degree $n$. 
In fact, let $P_{j}(f)=P_{j}, j=0, \ldots, m$, be the best uniform polynomial approximant of $f$ on $\left[x_{j-1}, x_{j+2}\right]$ of degree

$$
n_{j}:=\left[\frac{c_{1}}{800} n\left(\tilde{d}\left(x_{j}\right)\right)^{\gamma}\right] \text {, }
$$

and set

$$
P_{n}^{*}(x)=P(x):=\sum_{j=0}^{m} \chi_{j}(x) P_{j}(x) .
$$

Then $P$ is a polynomial of degree at most $n$ and below we show that $P_{n}^{*}=P$ satisfies the requirements set forth in Theorem 1.

Let $x \in\left[x_{k}, x_{k+1}\right]$ with $k$ arbitrary and let $j$ be different from $k-1, k$ and $k+1$. From (9) we get

$$
0 \leq \chi_{j}(x) \leq 2 C_{1} \exp \left[-\frac{c_{1}}{4} n\left|x-x_{j}\right|^{\gamma}\right],
$$

and the estimate (cf. $[4,2.13 .27])$

$$
|Q(y)| \leq(2|y|)^{\operatorname{deg} Q}\|Q\|_{[-1,1]}, \quad y \in \mathbf{R} \backslash[-1,1],
$$

transformed to the interval $\left[x_{j-1}, x_{j+2}\right]$ together with (5) and (6) easily implies

$$
\begin{aligned}
\left|f(x)-P_{j}(x)\right| & \leq\|f\|_{[0,1]}+\left(100\left|x-x_{j}\right| / \tilde{d}\left(x_{j}\right)\right)^{n_{j}}\left\|P_{j}\right\|_{\left[x_{j-1}, x_{j+2}\right]} \\
& \leq 3\|f\|_{[0,1]}\left(100\left|x-x_{j}\right| / \tilde{d}\left(x_{j}\right)\right)^{n_{j}} \\
& \leq 3\|f\|_{[0,1]} \exp \left[\frac{c_{1}}{800} n \tilde{d}\left(x_{j}\right)^{\gamma} \log \left(100\left|x-x_{j}\right| / \tilde{d}\left(x_{j}\right)\right)\right] \\
& \leq 3\|f\|_{[0,1]} \exp \left(\frac{c_{1}}{8} n e^{-1} \tilde{d}\left(x_{j}\right)^{\gamma-1}\left|x-x_{j}\right|\right) \\
& \leq 3\|f\|_{[0,1]} \exp \left(\frac{c_{1}}{8} n\left|x-x_{j}\right|^{\gamma}\right)
\end{aligned}
$$

because $100\left|x-x_{j}\right| / \tilde{d}\left(x_{j}\right)>1$ and $\log u \leq e^{-1} u$ for $u \geq 1$. Thus

$$
\begin{aligned}
\chi_{j}(x)\left|f(x)-P_{j}(x)\right| & \leq 6 C_{1}\|f\|_{[0,1]} \exp \left(-\frac{c_{1}}{8} n\left|x-x_{j}\right|^{\gamma}\right) \\
& \leq 6 C_{1}\|f\|_{[0,1]} \exp \left(-\frac{c_{1}}{16} n\left|x-x_{j}\right|^{\gamma}\right) \exp \left(-c_{2} n^{1-\gamma / \beta}\right) \\
& \leq 6 C_{1}\|f\|_{[0,1]} \exp \left(-c_{2} n d(x)^{\beta}\right) \exp \left(-c_{2} n^{1-\gamma / \beta}\right),
\end{aligned}
$$

where $c_{2}:=\left(c_{1} / 16\right) \cdot(1 / 150)>0$ and where we used that $\left|x-x_{j}\right| \geq(1 / 40) n^{-1 / \beta}$ (note that $x \notin\left[x_{j-1}, x_{j+2}\right]$ and $\left|x_{\ell+1}-x_{\ell}\right| \geq(1 / 40) n^{-1 / \beta}$ for any $0 \leq \ell \leq m$ ) and $\left|x-x_{j}\right| \geq(1 / 50) d(x)$. This immediately implies that for $x \in\left[x_{k}, x_{k+1}\right]$,

$$
\sum_{\substack{j=0 \\ j \neq k-1, k, k+1}}^{m} \chi_{j}(x)\left|f(x)-P_{j}(x)\right| \leq C_{2}\|f\|_{[0,1]} \exp \left(-c_{2} n[d(x)]^{\beta}\right)
$$

for some constant $C_{2}$ depending only on $\beta$. 
Since (cf. (8))

$$
f(x)-P(x)=\sum_{j=0}^{m} \chi_{j}(x)\left(f(x)-P_{j}(x)\right),
$$

we have to estimate $f(x)-P_{j}(x)$ for $j=k-1, k, k+1$, as well.

Again, let $x \in\left[x_{k}, x_{k+1}\right]$. If $d(x) \leq n^{-1 / \beta}$, then we just write

$$
\left|f(x)-P_{j}(x)\right| \leq\|f\|_{[0,1]} \leq e\|f\|_{[0,1]} \exp \left(-n[d(x)]^{\beta}\right) .
$$

If, however, $d(x)>n^{-1 / \beta}$, then $\tilde{d}(x) \leq 2 d(x)$, which, together with (7) yields

$$
\begin{aligned}
\left|f(x)-P_{j}(x)\right| & \leq E_{n_{j}}(f)_{\left[x_{k-2}, x_{k+3}\right]} \\
& \leq E_{n_{j}}(f)_{[x-\tilde{d}(x) / 4, x+\tilde{d}(x) / 4]} \\
& \leq E_{n_{j}}(f)_{[x-d(x) / 2, x+d(x) / 2]}
\end{aligned}
$$

where $E_{m}(f)_{[a, b]}$ denotes the error in best uniform approximation to $f$ on $[a, b]$ out of $\Pi_{m}$. To estimate the right-hand member of (13) consider the Taylor expansion of $f$ about $x$. For the absolute value of the $\nu$-th Taylor coefficient, Cauchy's inequality gives the bound

$$
\sup _{|z-x| \leq 3 d(x) / 4}|f(z)|\left(\frac{3}{4} d(x)\right)^{-\nu}=: C(x)\left(\frac{3}{4} d(x)\right)^{-\nu},
$$

and so the $n_{j}$-th partial sum approximates $f$ on $[x-d(x) / 2, x+d(x) / 2]$ with error at most

$$
C(x) \sum_{\nu=n_{j}+1}^{\infty}\left(\frac{2}{3}\right)^{\nu}=2 C(x) \exp \left(\left(\log \frac{2}{3}\right) n_{j}\right) \leq 2 C(x) \exp \left(-c_{3} n[d(x)]^{\beta}\right),
$$

with $c_{3}:=\left(\log \frac{3}{2}\right) c_{1} / 1000$ and where we used the fact that $n_{j}>\left(c_{1} / 1000\right) n[d(x)]^{\beta}$ whenever $n_{j} \geq N_{0}$ and $N_{0}$ satisfies $\left(c_{1} / 800\right) N_{0}^{1-\gamma / \beta} \geq 4$.

Thus

$$
E_{n_{j}}(f)_{[x-d(x) / 2, x+d(x) / 2]} \leq 2 C(x) \exp \left(-c_{3} n[d(x)]^{\beta}\right) .
$$

The relations (10)-(14) yield

$$
\begin{aligned}
\left|f(x)-P_{n}^{*}(x)\right| & \equiv|f(x)-P(x)| \\
& \leq C_{2}\|f\| \exp \left(-c_{2} n[d(x)]^{\beta}\right)+3\|f\| \leq C_{3}\|f\|
\end{aligned}
$$

and

$$
\left|f(x)-P_{n}^{*}(x)\right| \equiv|f(x)-P(x)| \leq C_{4}(x) \exp \left(-c_{3} n[d(x)]^{\beta}\right),
$$

which proves (3). Property (2) also can easily be obtained from (15). In fact, notice that every $n_{j}$ is at least as large as

$$
\frac{c_{1}}{1000} n(\tilde{d}(x))^{\gamma} \geq\left[\frac{c_{1}}{1000} n^{1-\gamma / \beta}\right]=: m_{n},
$$


and $m_{n} \rightarrow \infty$ as $n \rightarrow \infty$. If $Q_{m_{n}}$ denotes the best polynomial approximation of $f$ on $[0,1]$ by polynomials of degree at most $m_{n}$, then we get from $n_{j} \geq m_{n}$

$$
P_{j}(f ; \cdot)_{\left[x_{j-1}, x_{j+2}\right]}-Q_{m_{n}}(\cdot) \equiv P_{j}\left(f-Q_{m_{n}} ; \cdot\right)_{\left[x_{j-1}, x_{j+2}\right]},
$$

and so (cf. (8))

$$
f(\cdot)-\sum_{j=0}^{m} \chi_{j}(\cdot) P_{j}(f ; \cdot) \equiv\left(f-Q_{m_{n}}\right)(\cdot)-\sum_{j=0}^{m} \chi_{j}(\cdot) P_{j}\left(f-Q_{m_{n}} ; \cdot\right) .
$$

Hence we obtain from (15), with $f$ replaced by $f-Q_{m_{n}}$ on the right, that

$$
\left|f(x)-P_{n}^{*}(x)\right| \leq C_{3}\left\|f-Q_{m_{n}}\right\|_{[0,1]} .
$$

Since $\left\|f-Q_{m_{n}}\right\|_{[0,1]} \rightarrow 0$ as $n \rightarrow \infty$, we obtain (2).

\section{Proof of Theorem 2}

Suppose to the contrary that for any $a \in(0,1 / 2]$ the estimate

$$
\left|P_{n}(x)-\right| x|| \leq C_{a} \exp (-2 c n|x|), \quad x \in[-1,1] \backslash[-a, a],
$$

is possible with some positive constants $C_{a}$ and $c$ and polynomials $P_{n} \in \Pi_{n}$. Then (16) holds for $\left(P_{n}(x)+P_{n}(-x)\right) / 2$, as well, so we may assume each $P_{n}$ to be even.

Consider the derivative $P_{n}^{\prime}$ of $P_{n}$, which is odd. Let $x \in[a, 1]$ and $b:=$ $\min \{x, 1 / 2\}$. Since

$$
\left|P_{n}(u)-u\right| \leq C_{a} \exp (-2 c n b)
$$

if $u \in[b, 1]$, we get from Markoff's inequality, applied to the interval $[b, 1]$, that

$$
\begin{aligned}
\left|P_{n}^{\prime}(x)-1\right| & \leq 4 C_{a} n^{2} \exp (-2 c n b) \\
& \leq 4 C_{a} n^{2} \exp (-c n x), \quad a \leq x \leq 1
\end{aligned}
$$

In a similar way

$$
\left|P_{n}^{\prime}(x)+1\right| \leq 4 C_{a} n^{2} \exp (-c n|x|), \quad-1 \leq x \leq-a,
$$

and so for $a \leq|x| \leq 1$ we have

$$
\left|P_{n}^{\prime}(x)-\operatorname{sign} x\right| \leq 4 C_{a} n^{2} \exp (-c n|x|),
$$

where $C_{a}$ is independent of $n$.

This implies that the polynomials

$$
Q_{n}(x):=1-\left(P_{n}^{\prime}(\sqrt{x})\right)^{2}
$$

of degree at most $n$ satisfy $Q_{n}(0)=1$ and, for large $n$,

$$
\left|Q_{n}(x)\right| \leq 8 C_{a} n^{2} \exp (-c n \sqrt{x}), \quad a \leq x \leq 1 .
$$


However, this is impossible, since in [1, Section 4.I] it was proved that if $Q_{n} \in$ $\Pi_{n}$ and $Q_{n}(0)=1$, then for $0<a \leq 1$ the inequality $\left|Q_{n}(x)\right| \leq 1$ on $[a, 1]$ implies

$$
\int_{a}^{1} \frac{-\log \left|Q_{n}(x)\right|}{x^{3 / 2}} d x \leq 4 n .
$$

This contradiction proves the theorem.

\section{Proof Theorem 3}

Let $a_{k}:=2^{-k}(1+i)$, where $i=\sqrt{-1}$, and set

$$
f(z):=\sum_{k=1}^{\infty} n_{k}^{-1 / 8}\left[2^{k}\left(z-a_{k}\right)\right]^{-n_{k}}=: \sum_{k=1}^{\infty} g_{k}(z),
$$

where $\left\{n_{k}\right\}$ will be completely specified later in the proof. We set $n_{1}=1$, and require $n_{k+1}>2^{k} n_{k}$ for all $k$ so that for every $\ell>0$ we have

$$
n_{k} / 2^{\ell k} \rightarrow \infty \text {. }
$$

Since

$$
\left|\left[2^{k}\left(z-a_{k}\right)\right]^{-1}\right| \leq 1 \quad \text { if }\left|z-a_{k}\right| \geq 2^{-k},
$$

it is easy to see that the series defining $f$ uniformly converges on the real line and also on every compact subset of the complex plane not containing the points $0,\left\{a_{k}\right\}_{k=1}^{\infty}$; thus these are the singular points of $f$. In particular,

$$
d(x) \geq \frac{1}{\sqrt{2}} x \quad \text { for } x \in[0,1] .
$$

Now we need some easy estimates:

$$
\begin{aligned}
\left|g_{k}\left(2^{-k}\right)\right| & =n_{k}^{-1 / 8} \\
\left|g_{k}\left(2^{-k}+n_{k}^{-1 / 4}\right)\right| & =n_{k}^{-1 / 8}\left(1+4^{k} n_{k}^{-1 / 2}\right)^{-n_{k} / 2}<\frac{1}{2} n_{k}^{-1 / 8}
\end{aligned}
$$

and so

$$
n_{k}^{1 / 4}\left|g_{k}\left(2^{-k}\right)-g_{k}\left(2^{-k}+n_{k}^{-1 / 4}\right)\right|>\frac{1}{2} n_{k}^{1 / 8} .
$$

Using this, by standard gliding-hump arguments we can select $n_{1}, n_{2}, \ldots$ one after the other in such a way that

$$
n_{k}^{1 / 4}\left|f\left(2^{-k}\right)-f\left(2^{-k}+n_{k}^{-1 / 4}\right)\right|>\frac{1}{4} n_{k}^{1 / 8}
$$

is satisfied for all $k$.

Now if (4) is true for some $C, c>0$ then

(20) $\left|f(x)-P_{m}(x)\right| \leq 2^{k} \exp \left(-2^{-k} m[d(x)]^{\beta}\right), \quad m=1,2, \ldots, \quad x \in[0,1]$, is also true for any large $k, k \geq \max \{\ln C ;-\ln c\} / \ln 2$, and below we show that this is impossible. 
Fix a $k$ so large that $(20)$ is satisfied and also $(1 / 16) n_{k}^{-1 / 8}<2^{k} \exp$ $\left\{-2^{-\beta / 2} n_{k}^{1 / 16}\right\}$, which is always possible because of (17).

Set $m=\left[2^{k(1+\beta)} n_{k}^{1 / 16}\right]+1$ into (20). Then for $x \in\left[2^{-k}, 1\right]$ we get from (18) and $(20)$

$$
\left|f(x)-P_{m}(x)\right| \leq 2^{k} \exp \left(-2^{-k} 2^{k(1+\beta)} n_{k}^{1 / 16}\left(2^{-k} / \sqrt{2}\right)^{\beta}\right)<\frac{1}{16} n_{k}^{-1 / 8} ;
$$

therefore (cf. (19)),

$$
n_{k}^{1 / 4}\left|P_{m}\left(2^{-k}\right)-P_{m}\left(2^{-k}+n_{k}^{-1 / 4}\right)\right|>\frac{1}{8} n_{k}^{1 / 8} .
$$

On the other hand, $\left\{P_{m}\right\}$ are uniformly bounded on $[0,1]$, say $\left|P_{m}\right| \leq K$, $m=1,2, \ldots$, (cf. (4)); hence Bernstein's inequality shows that for $x \in$ $\left[2^{-k}, 2^{-k}+n_{k}^{-1 / 4}\right]$,

$$
\left|P_{m}^{\prime}(x)\right| \leq K m 2^{k}
$$

which implies

$$
\begin{aligned}
n_{k}^{1 / 4}\left|P_{m}\left(2^{-k}\right)-P_{m}\left(2^{-k}+n_{k}^{-1 / 4}\right)\right| & \leq K m 2^{k} \\
& \leq 2 K 2^{k(2+\beta)} n_{k}^{1 / 16} .
\end{aligned}
$$

However, (17), (21) and (22) are not compatible which proves our theorem.

\section{REFERENCES}

1. K. G. Ivanov and V. Totik, Fast decreasing polynomials, Constr. Approx., 5 (1989).

2. M. I. Kadec, On the distribution of maximum deviation in the approximation of continuous functions, Amer. Math. Soc. Transl., 26 (1963), 231-234.

3. E. B. Saff and V. Totik, Polynomial approximation of piecewise analytic functions, Journal London Math. Soc., (to appear).

4. A. F. Timan, Theory of approximation of functions of a real variable, Pergamon Press, New York, 1963.

Institute of Mathematics, Bulgarian ACAdemy of Sciences, Sofia, 1090, Bulgaria

Institute for Constructive Mathematics, University of South Florida, Tampa, FLORIDA 33620

Bolyai Institute, Aradi V. Tere 1, Szeged, 6720, Hungary 